NISSUNA UMANA INVESTIGAZIONE SI PUO DIMANDARE VERA SCIENZIA S'ESSA NON PASSA PER LE MATEMATICHE DIMOSTRAZIONI LEONARDO DA VINCI

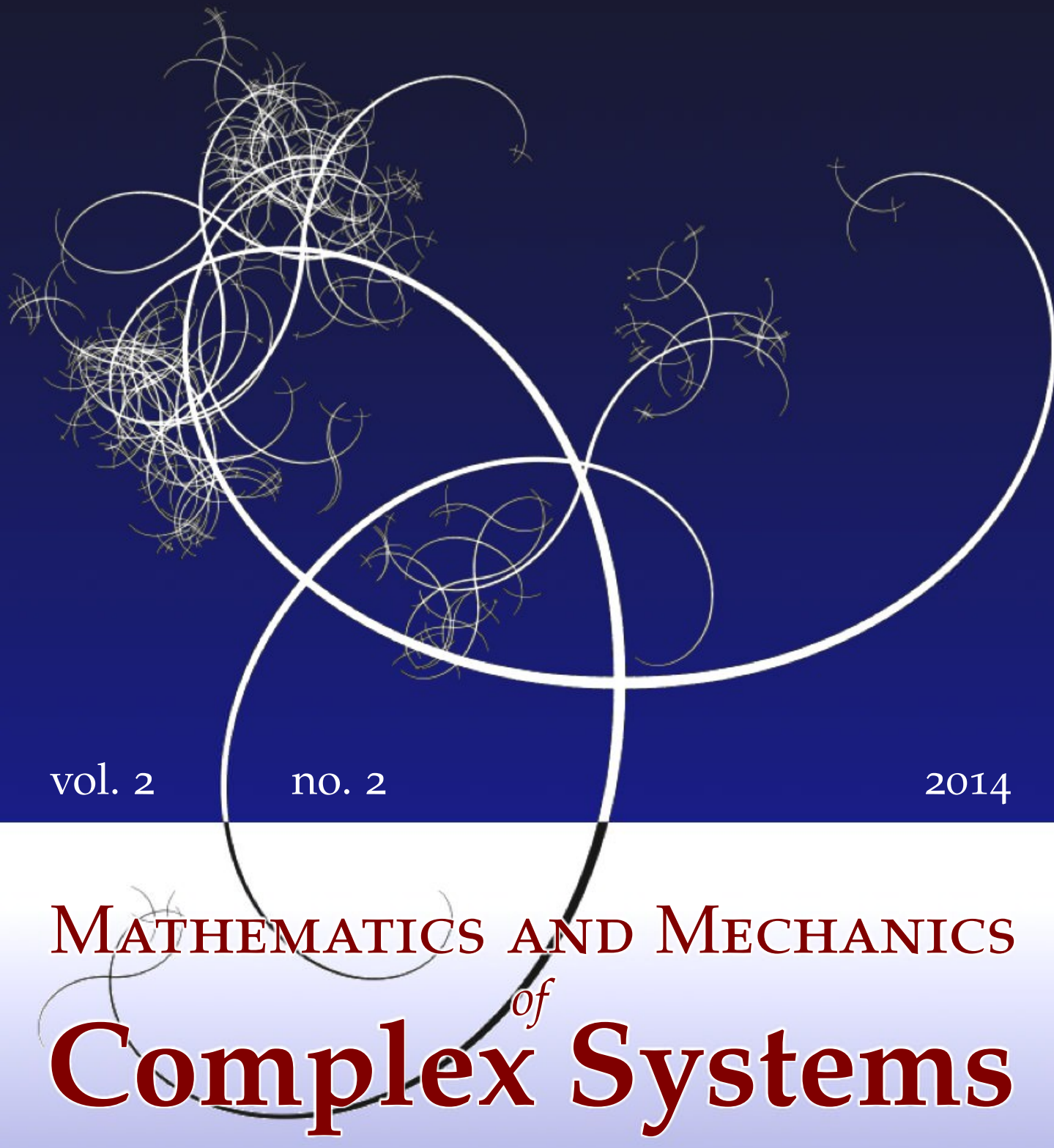

ANATOLIY MALYARENKO AND MARTIN OSTOJA-STARZEWSKI STATISTICALLY ISOTROPIC TENSOR RANDOM FIELDS: CORRELATION STRUCTURES 


\title{
STATISTICALLY ISOTROPIC TENSOR RANDOM FIELDS: CORRELATION STRUCTURES
}

\author{
Anatoliy Malyarenko AND Martin OStojA-STARZEWSKi
}

Let $V$ be a real finite-dimensional vector space. We introduce some physical problems that may be described by $V$-valued homogeneous and isotropic random fields on $\mathbb{R}^{3}$. We propose a general method for calculation of expectations and two-point correlation functions of such fields. Our results are equivalent to classical results by Robertson, when $V=\mathbb{R}^{3}$, and those by Lomakin, when $V$ is the space of symmetric second-rank tensors over $\mathbb{R}^{3}$. Our solution involves an analogue of the classical Clebsch-Gordan coefficients.

\section{Introduction}

The entire field of continuum physics involves tensor fields. Overwhelmingly, most of the existing models and theories are deterministic and their stochastic generalizations necessitate construction of tensor-valued random fields (RF). While the literature on scalar RFs is vast (for example, [Cressie 1993; Christakos 2005; Marinucci and Peccati 2011; Leonenko and Sakhno 2012; Porcu et al. 2012]), that on vector RFs is largely limited to statistical turbulence [Monin and Yaglom 1965], and the case of higher tensor rank (second, fourth) RFs poses challenges. In this paper we focus on wide-sense stationary and statistically isotropic RFs of tensors of the first and second ranks. We present a new method of derivation of representations of their correlation functions, which in the case of first-rank tensors gives the same result as in [Robertson 1940], while in the case of second-rank tensors is equivalent to the result of [Lomakin 1964].

These representations have applications to tensor random fields (TRFs) governed by the field equations of continuum physics as well as those representing some spatially inhomogeneous constitutive properties of random media. The former type of TRFs is used in [Ostoja-Starzewski et al. 2013], where correlation functions are subject to constraints such as the equilibrium equation or straindisplacement relation. The basic properties of TRFs of a wide-sense homogeneous and isotropic kind with generally anisotropic realizations have been determined

\section{Communicated by Eric A. Carlen.}

MSC2010: primary 60G60; secondary 74A40.

Keywords: isotropic tensor random field, group representation, Godunov-Gordienko coefficients. 
in three continuum physics theories: thermal conduction, classical elasticity, and micropolar elasticity. The field equations (such as the linear and angular momentum balances and strain-displacement relations), all in a quasistatic setting, lead to consequences for the respective dependent fields involved. In effect, these consequences are restrictions on the admissible forms of the correlation functions describing the TRFs.

The latter type of TRFs provides models of random media described by the second-rank TRF. The typical example here is the thermal conductivity tensor and its mathematical analogies such as the antiplane stiffness tensor. Once the general representation of this TRF is established and the conditions of positive definiteness are imposed, one can turn to modeling and simulation of the entire range of statistical constitutive behaviors of all heat-conducting media or, say, elastic materials subjected to antiplane loading, for example, [Sena et al. 2013].

In particular, let $V$ be a finite-dimensional real Hilbert space with norm $\|\cdot\|$. Let $\boldsymbol{T}(\boldsymbol{x}), \boldsymbol{x} \in \mathbb{R}^{3}$, be a random field taking values in (a subset of) $V$. Suppose that $\mathrm{E}\left[\|\boldsymbol{T}(\boldsymbol{x})\|^{2}\right]<\infty$ and that $\boldsymbol{T}(\boldsymbol{x})$ is mean-square continuous, that is, for any $\boldsymbol{x}_{0} \in \mathbb{R}^{3}$ we have

$$
\lim _{\left\|\boldsymbol{x}-\boldsymbol{x}_{0}\right\| \rightarrow 0} \mathrm{E}\left[\left\|\boldsymbol{T}(\boldsymbol{x})-\boldsymbol{T}\left(\boldsymbol{x}_{0}\right)\right\|^{2}\right]=0 .
$$

Let $E(\boldsymbol{x})=\mathrm{E}[\boldsymbol{T}(\boldsymbol{x})]$ be the expectation of the field, and let $B(\boldsymbol{x}, \boldsymbol{y})=\mathrm{E}[\boldsymbol{T}(\mathbf{x}) \otimes$ $\boldsymbol{T}(\boldsymbol{y})]$ be the two-point correlation function of the random field $\boldsymbol{T}(\boldsymbol{x})$. The group $\mathbb{R}^{3}$ acts on itself by translations. Assume that the above functions are invariant with respect to this action, that is, for all $\boldsymbol{x}, \boldsymbol{y}, \boldsymbol{z} \in \mathbb{R}^{3}$,

$$
\begin{aligned}
E(x+z) & =E(x), \\
B(x+z, y+z) & =B(x, y) .
\end{aligned}
$$

It follows that $E(\boldsymbol{x})=\boldsymbol{E} \in V$ is constant, while $B(\boldsymbol{x}, \boldsymbol{y}) \in V \otimes V$ depends only on the difference $\boldsymbol{x}-\boldsymbol{y}$.

Let $K=\mathrm{SO}(3)$ be the group of rotations in $\mathbb{R}^{3}$, and let $(V, \gamma)$ be an orthogonal representation of $K$. Suppose that for all $k \in K$ and all $x \in \mathbb{R}^{3}$ we have

$$
\begin{aligned}
& E(k \boldsymbol{x})=\gamma(k) E(\boldsymbol{x}), \\
& B(k \boldsymbol{x})=\gamma(k) B(\boldsymbol{x}) \gamma^{-1}(k) .
\end{aligned}
$$

We would like to find a general form for the expectation and two-point correlation function of such a field.

In Section 2, we consider mathematical preliminaries. We use the book [Adams 1969], in which Adams considers both real and complex representations at the same time.

In Section 3 we consider two particular cases of the above problem: 
(1) $V$ has dimension 3 and $\gamma(k)=k$.

(2) $V$ is the space of all second-rank tensors over $\mathbb{R}^{3}, \boldsymbol{T}(\boldsymbol{x})$ takes values in the set of all symmetric tensors, and the representation is $\gamma(k) \boldsymbol{T}=k \boldsymbol{T} k^{-1}$.

In the first case, the answer has been known since the classic paper [Robertson 1940]. In Theorem 3.1, we prove that our method of solution gives the same answer. Our new result is Theorem 3.2. Section 4 concludes. Proofs of our results are collected in the Appendix.

\section{Mathematical preliminaries}

Let $\mathbb{K}$ be either the field $\mathbb{R}$ of real numbers or the field $\mathbb{C}$ of complex numbers, and let $K$ be a topological group with the identity element $e$. A representation of the group $K$ over $\mathbb{K}$ is a pair $(V, \gamma)$, where $V$ is a finite-dimensional vector space over $\mathbb{K}$, and $\gamma$ is a continuous homomorphism from $K$ to the group Aut $V$ of the invertible linear operators in $V$. In other words, for each $k \in K$ and for each $v \in V$ there is a vector $\gamma(k) v \in V$, and the following conditions hold true:

(1) $\gamma(e) \boldsymbol{v}=\boldsymbol{v}$ and $\gamma(k)\left(\gamma\left(k^{\prime}\right) \boldsymbol{v}\right)=\gamma\left(k k^{\prime}\right) \boldsymbol{v}$.

(2) $\gamma(k) \boldsymbol{v}$ is a $\mathbb{K}$-linear function of $\boldsymbol{v}$.

(3) $\gamma(k) v$ is a continuous function of $k$ and $\boldsymbol{v}$.

Let $(V, \gamma)$ and $(W, \delta)$ be two representations. A map $G: V \rightarrow W$ is called a $K$-map if

$$
G(\gamma(k) v)=\delta(k)(G \boldsymbol{v}) .
$$

A $\mathbb{K}$-linear $K$-map is called an intertwining operator. The set of all intertwining operators is a vector space over $\mathbb{K}$. The representations $(V, \gamma)$ and $(W, \delta)$ are called equivalent if the above space contains an invertible operator.

Let $(V, \gamma)$ be a real representation of the group $K$. Build a complex representation $\left(V^{\prime}, \gamma^{\prime}\right)$ as follows. Consider $\mathbb{C}$ as a vector space over $\mathbb{R}$. Put $V^{\prime}=\mathbb{C} \otimes_{\mathbb{R}} V$. The space $V^{\prime}$ is a complex vector space, where multiplication by a complex number $z$ is defined as $z\left(z^{\prime} \otimes \boldsymbol{v}\right)=z z^{\prime} \otimes \boldsymbol{v}$. The representation $\gamma^{\prime}$ is

$$
\gamma^{\prime}(k)(z \otimes \boldsymbol{v})=z \otimes \gamma(k) \boldsymbol{v} .
$$

Define a map $j: V^{\prime} \rightarrow V^{\prime}$ by $j(z \otimes v)=\bar{z} \otimes \boldsymbol{v}$. Then $j$ is a structural map, that is, a $K$-map with

$$
j(z \boldsymbol{v})=\bar{z} j(\boldsymbol{v}), \quad j^{2}=1 .
$$

Conversely, let $\left(V^{\prime}, \gamma^{\prime}\right)$ be a complex representation of $K$ that admits a structural map $j$. Then $V^{\prime}$ is a direct sum of two eigenspaces $V_{+}$and $V_{-}$of the map $j$ that correspond to the eigenvalues +1 and -1 . These spaces carry two equivalent real 
representations. Multiplication by $\mathrm{i}$ is an invertible intertwining operator between the above representations.

The direct sum of two representations $(V, \gamma)$ and $(W, \delta)$ is the representation $(V \oplus W, \gamma \oplus \delta)$, where

$$
\gamma \oplus \delta(k)(\boldsymbol{v} \oplus \boldsymbol{w})=(\gamma(k) \boldsymbol{v}) \oplus(\delta(k) \boldsymbol{w}) .
$$

The tensor product of two representations $(V, \gamma)$ and $(W, \delta)$ is the representation $(V \otimes W, \gamma \otimes \delta)$, where

$$
\gamma \otimes \delta(k)(\boldsymbol{v} \otimes \boldsymbol{w})=(\gamma(k) \boldsymbol{v}) \otimes(\delta(k) \boldsymbol{w}) .
$$

A representation $(V, \gamma)$ with $V \neq\{\mathbf{0}\}$ is called reducible if there exists a proper subspace $W$ of $V$ with $\gamma(k) \boldsymbol{w} \in W$ for all $\boldsymbol{w} \in W$ and $k \in K$ and irreducible otherwise. If $K$ is a compact group, then any representation $(V, \gamma)$ of $K$ is a direct sum of irreducible representations. Moreover, the decomposition onto irreducible representations is unique in the following sense. If $m_{i} V_{i}$ denotes the direct sum of $m_{i}$ copies of the representation $V_{i}$, and the representations $\bigoplus m_{i} V_{i}$ and $\bigoplus n_{i} V_{i}$ are equivalent, then $m_{i}=n_{i}$ for all $i$.

Let $(V, \gamma)$ be a complex representation of a compact topological group $K$. By [Adams 1969, Proposition 3.16], there exists a $K$-invariant inner product $(\cdot, \cdot)$ on $V$. Moreover, if $(V, \gamma)$ admits a structural map $j$, one can choose the above inner product in such a way that $(j \boldsymbol{v}, j \boldsymbol{w})=\overline{(\boldsymbol{v}, \boldsymbol{w})}$, and the restriction of the inner product to either space $V_{+}$or $V_{-}$is again an inner product.

Choose an orthonormal basis $\boldsymbol{e}_{1}, \ldots, \boldsymbol{e}_{n}$ in $V$. Then, the complex representation $\gamma$ takes values in the unitary group $U(n)$ and is called a unitary representation. A real representation takes values in the orthogonal group $O(n)$ and is called an orthogonal representation.

Realize $\mathbb{R}^{3}$ as the space of traceless Hermitian matrices in $\mathbb{C}^{2}$. Such a matrix has the form

$$
A=\left(\begin{array}{cc}
x_{0} & x_{1}+x_{-1} \mathrm{i} \\
x_{1}-x_{-1} \mathrm{i} & -x_{0}
\end{array}\right), \quad x_{-1}, x_{0}, x_{1} \in \mathbb{R} .
$$

The map $A \mapsto k^{-1} A k$, where $k$ is an element of the group SU(2) of unitary $2 \times 2$ matrices with unit determinant, is a rotation, that is, an element of the group $\mathrm{SO}(3)$. The matrices $k$ and $-k$ determine the same rotation. Conversely, each rotation in $\mathrm{SO}(3)$ corresponds to a pair of matrices $k$ and $-k$.

Let $(V, \gamma)$ be an irreducible unitary representation of the group $\mathrm{SU}(2)$. If $\gamma(k)=$ $\gamma(-k)$, then $(V, \gamma)$ is an irreducible unitary representation of the group $\operatorname{SO}(3)$, and all irreducible unitary representations of $\mathrm{SO}(3)$ may be obtained in this way. 
Let

$$
k=\left(\begin{array}{rr}
\alpha & \beta \\
-\bar{\beta} & \bar{\alpha}
\end{array}\right), \quad \alpha, \beta \in \mathbb{C}, \quad|\alpha|^{2}+|\beta|^{2}=1,
$$

be an element of the group SU(2). Let $V^{\ell}$ be the space of homogeneous polynomials of degree $2 \ell$ in two complex variables $\xi$ and $\eta$. The representation

$$
\gamma_{\ell}(k) f(\xi, \eta)=f(\bar{\alpha} \xi-\beta \eta, \bar{\beta} \xi+\alpha \eta)
$$

is irreducible. Conversely, any irreducible representation of the group $\mathrm{SU}(2)$ is equivalent to the representation (2-1).

If $\ell$ is an integer, then $\gamma_{\ell}(k)=\gamma_{\ell}(-k)$, and the representation (2-1) is an irreducible representation of the group $\mathrm{SO}(3)$. Moreover, put

$$
j f(\xi, \eta)=\bar{f}(-\eta, \xi)
$$

where $\bar{f}$ is the polynomial with coefficients which are complex conjugate to those of $f$. Then $j$ is a structural map. If we choose an orthonormal basis $\boldsymbol{e}_{m}^{\ell},-\ell \leq m \leq \ell$, satisfying the condition

$$
j \boldsymbol{e}_{m}^{\ell}=\boldsymbol{e}_{m}^{\ell},
$$

then the restriction $\left(V_{+}^{\ell}, \gamma_{\ell,+}\right)$ of the representation $\left(V^{\ell}, \gamma_{\ell}\right)$ to the real linear span $V_{+}^{\ell}$ of the above basis is an irreducible real representation, and the matrix entries of the operators $\gamma_{\ell}(k)$ are real-valued functions on the group $\mathrm{SO}(3)$. If the basis $\boldsymbol{e}_{m}^{\ell}$ satisfies the condition

$$
j \boldsymbol{e}_{m}^{\ell}=-\boldsymbol{e}_{m}^{\ell}
$$

then the restriction $\left(V_{-}^{\ell}, \gamma_{\ell,-}\right)$ of the representation $\left(V^{\ell}, \gamma_{\ell}\right)$ to the real linear span $V_{-}^{\ell}$ of the above basis is an irreducible real representation, equivalent to $\left(V_{+}^{\ell}, \gamma_{\ell,+}\right)$, and multiplication by $\mathrm{i}$ is an orthogonal intertwining operator between two equivalent representations.

The usual orthonormal basis in the space $V^{\ell}$ is as follows:

$$
\boldsymbol{f}_{m}^{\ell}(\xi, \eta)=(-1)^{\ell+m} \sqrt{\frac{(2 \ell+1) !}{(\ell+m) !(\ell-m) !}} \xi^{\ell+m} \eta^{\ell-m}
$$

The matrix entries of operators $\gamma_{\ell}(k)$ in this basis are called the Wigner D-functions and are denoted by $D_{m n}^{\ell}(k)$. The basis (2-4) does not satisfy (2-2). Gordienko 
[2002] proposed the basis satisfying (2-2) as follows:

$$
\begin{aligned}
\boldsymbol{h}_{-m}^{\ell}(\xi, \eta) & =\frac{(-\mathrm{i})^{\ell-1}}{\sqrt{2}}\left[(-1)^{m} \boldsymbol{f}_{m}^{\ell}(\xi, \eta)-\boldsymbol{f}_{-m}^{\ell}(\xi, \eta)\right], \\
\boldsymbol{h}_{0}^{\ell}(\xi, \eta) & =(-\mathrm{i})^{\ell} \boldsymbol{e}_{0}^{\ell}(\xi, \eta), \\
\boldsymbol{h}_{m}^{\ell}(\xi, \eta) & =-\frac{(-\mathrm{i})^{\ell}}{\sqrt{2}}\left[(-1)^{m} \boldsymbol{f}_{m}^{\ell}(\xi, \eta)+\boldsymbol{f}_{-m}^{\ell}(\xi, \eta)\right],
\end{aligned}
$$

where $m \geq 1$. From now on, we define by $U^{\ell}(k)$ the matrices of the representation $\left(V_{+}^{\ell}, \gamma_{\ell,+}\right)$ in the Gordienko basis and omit + and - for simplicity of notation. Note that $\mathrm{i} \boldsymbol{h}_{m}^{\ell}(\xi, \eta)$ is the Gordienko basis of the space $V_{-}$. Its vectors satisfy (2-3).

Any rotation $k$ may be performed by three successive rotations:

- rotation $k_{0}(\psi)$ about the $x_{0}$-axis through an angle $\psi, 0 \leq \psi<2 \pi$,

- rotation $k_{-1}(\theta)$ about the $x_{-1}$-axis through an angle $\theta, 0 \leq \theta \leq \pi$, and

- rotation $k_{0}(\varphi)$ about the $x_{0}$-axis through an angle $\varphi, 0 \leq \varphi<2 \pi$.

The angles $\psi, \theta$, and $\varphi$ are the Euler angles. The map which maps the product of the above rotations $k(\psi, \theta, \varphi)$ to the point $(\psi, \theta, \varphi) \in(0,2 \pi) \times(0, \pi) \times(0,2 \pi)$ is a chart of the group manifold $\mathrm{SO}(3)$, and the domain of this chart is an open dense subset of $\mathrm{SO}(3)$. Moreover, the map which maps the rotation $k(0, \theta, \varphi)(0,1,0)^{\top}$ to the point $(\theta, \varphi) \in(0, \pi) \times(0,2 \pi)$ is a chart of the unit sphere $S^{2}$ centered at the origin of the space $\mathbb{R}^{3}$. The coordinates of the point $k(0, \theta, \varphi)(0, r, 0)^{\top}, r>0$, are the spherical coordinates,

$$
\begin{aligned}
x_{-1} & =r \sin \varphi \sin \theta, \\
x_{0} & =r \cos \theta, \\
x_{1} & =r \cos \varphi \sin \theta,
\end{aligned}
$$

Gordienko [2002] calculated the matrix entries of the matrices $U^{\ell}(k)$. His result is as follows. If $k=k(\psi, \theta, \varphi)$, then

$$
U^{\ell}(k)=U^{\ell}\left(k_{0}(\varphi)\right) U^{\ell}\left(k_{-1}(\theta)\right) U^{\ell}\left(k_{0}(\psi)\right),
$$

by the definition of a representation. Denote the matrix entries of the matrix $U^{\ell}\left(k_{0}(\varphi)\right)$ by $\Omega_{0, m, n}^{\ell}(\varphi)$, where $-\ell \leq m, n \leq \ell$. The nonzero entries are

$$
\Omega_{0,0,0}^{\ell}(\varphi)=1, \quad \Omega_{0, m, m}^{\ell}(\varphi)=\cos (m \varphi), \quad \Omega_{0,-m, m}^{\ell}(\varphi)=\sin (m \varphi),
$$


where $m= \pm 1, \pm 2, \ldots, \pm \ell$. Denote the matrix entries of the matrix $U^{\ell}\left(k_{-1}(\theta)\right)$ by $\Omega_{-1, m, n}^{\ell}(\theta)$. The nonzero entries are

$$
\begin{aligned}
\Omega_{-1, \pm m, \pm n}^{\ell}(\theta)= & \frac{(-1)^{\ell-n}}{2^{\ell}\left(1-\mu^{2}\right)^{n / 2}} \sqrt{\frac{(\ell+n) !}{(\ell-n) !(\ell-m) !(\ell+m) !}} \\
& \times\left\{\left(\frac{1-\mu}{1+\mu}\right)^{n / 2} \frac{\mathrm{d}^{\ell-n}}{\mathrm{~d} \mu^{\ell-n}}\left[(1+\mu)^{\ell+m}(1-\mu)^{\ell-m}\right]\right. \\
& \left. \pm(-1)^{m}\left(\frac{1+\mu}{1-\mu}\right)^{n / 2} \frac{\mathrm{d}^{\ell-n}}{\mathrm{~d} \mu^{\ell-n}}\left[(1+\mu)^{\ell-m}(1-\mu)^{\ell+m}\right]\right\} \\
\Omega_{-1,0,0}^{\ell}(\theta)= & \frac{(-1)^{\ell}}{2^{\ell} \ell !} \frac{\mathrm{d}^{\ell}}{\mathrm{d} \mu^{\ell}}\left(1-\mu^{2}\right)^{\ell} \\
\Omega_{-1,0, n}^{\ell}(\theta)= & \frac{(-1)^{\ell-n}}{2^{\ell} \ell !} \sqrt{\frac{2(\ell+n) !}{(\ell-n) !}} \frac{1}{\left(1-\mu^{2}\right)^{n / 2}} \frac{\mathrm{d}^{\ell-n}}{\mathrm{~d} \mu^{\ell-n}}\left(1-\mu^{2}\right)^{\ell} \\
\Omega_{-1, m, 0}^{\ell}(\theta)=- & \frac{(-1)^{\ell}}{2^{\ell} \ell !} \sqrt{\frac{2(\ell+m) !}{(\ell-m) !}} \frac{1}{\left(1-\mu^{2}\right)^{m / 2}} \frac{\mathrm{d}^{\ell-m}}{\mathrm{~d} \mu^{\ell-m}}\left(1-\mu^{2}\right)^{\ell}
\end{aligned}
$$

where $m \geq 1, n \geq 1$, and where $\mu=\cos \theta$.

Let $\left(V^{m}, \gamma_{m}\right)$ and $\left(V^{p}, \gamma_{p}\right)$ be two irreducible orthogonal representations of the group $\mathrm{SO}(3)$. Their tensor product $\left(V^{m} \otimes V^{p}, \gamma_{m} \otimes \gamma_{p}\right)$ is equivalent to the direct $\operatorname{sum}\left(V^{|m-p|} \oplus V^{|m-p|+1} \oplus \cdots \oplus V^{m+p}, \gamma_{|m-p|} \oplus \gamma_{|m-p|+1} \oplus \cdots \oplus \gamma_{m+p}\right)$. Let $G$ be the orthogonal intertwining operator between the above equivalent representations. Then we have

$$
G\left(\gamma_{m}(k) \otimes \gamma_{p}(k)\right)=\left(\gamma_{|m-p|(k)} \oplus \gamma_{|m-p|+1}(k) \oplus \cdots \oplus \gamma_{m+p}(k)\right) G .
$$

In the usual basis (2-4), this equality takes the form

$$
\sum_{i=-m}^{m} \sum_{j=-p}^{p} C_{m i p j}^{s \ell} D_{n i}^{m}(k) D_{q j}^{p}(k)=\sum_{t=-s}^{s} U_{t \ell}^{s}(k) C_{m n p q}^{s t},
$$

which is [Varshalovich et al. 1975, Equation (5), §4.6]. The matrix entries of the operator $G$ in the basis (2-4), $C_{m i p j}^{s \ell}$, are called the Clebsch-Gordan coefficients.

In the Gordienko basis, the same equality takes the form

$$
\sum_{i=-m}^{m} \sum_{j=-p}^{p} g_{s[m, p]}^{\ell[i, j]} U_{n i}^{m}(k) U_{q j}^{p}(k)=\sum_{t=-s}^{s} U_{t \ell}^{s}(k) g_{s[m, p]}^{t[n, q]} .
$$

We call the matrix entries of the operator $G$ in the Gordienko basis, $g_{s[m, p]}^{\ell[i, j]}$, the 
Godunov-Gordienko coefficients. They were calculated in [Godunov and Gordienko 2004].

It follows in particular that the matrix $G$ transforms the uncoupled basis $\boldsymbol{h}_{t}^{s}$, $|m-p| \leq s \leq m+p,-s \leq t \leq s$, to the coupled basis $\boldsymbol{h}_{n}^{m} \otimes \boldsymbol{h}_{q}^{p}$ :

$$
\boldsymbol{h}_{n}^{m} \otimes \boldsymbol{h}_{q}^{p}=\sum_{r=|m-p|}^{m+p} \sum_{s=-r}^{r} g_{r[m, p]}^{s[n, q]} \boldsymbol{h}_{s}^{r}
$$

We multiply both sides of (2-8) by $G^{-1}$ from the left and write the result in the Gordienko basis. We obtain

$$
U_{n i}^{m}(k) U_{q j}^{p}(k)=\sum_{s=|m-p|}^{m+p} \sum_{t=-s}^{s} \sum_{\ell=-s}^{s} g_{s[m, p]}^{t[n, q]} U_{t \ell}^{s}(k) g_{s[m, p]}^{\ell[i, j]} .
$$

The same equality in the usual basis is [Varshalovich et al. 1975, Equation (1), $\S 4.6]$. It is called the Clebsch-Gordan expansion.

Lemma 2.1 [Malyarenko 2013]. Let $U$ be an irreducible representation of a topological group $K$ in a Hilbert space $H$. Let $\boldsymbol{x} \in H$ be a common eigenvector of all operators $U(k), g \in K$. If $U$ is not trivial, then $\boldsymbol{x}=\mathbf{0}$.

Lemma 2.2. The second equation in (1-1) may be written in the Gordienko basis as follows:

$$
B(k \boldsymbol{x})=(U \otimes U)(k) B(\boldsymbol{x})
$$

\section{The results}

Theorem 3.1 [Robertson 1940]. Let $\boldsymbol{T}(\boldsymbol{x})$ be a $V$-valued random field on $\mathbb{R}^{3}$ satisfying (1-1) with $U(k)=k$. Then

$$
\mathrm{E}[\boldsymbol{T}(\boldsymbol{x})]=\mathbf{0}
$$

and there exist two continuous functions $K_{0}, K_{2}:[0, \infty) \rightarrow \mathbb{R}$ with $K_{2}(0)=0$ such that

$$
B_{i j}(\boldsymbol{x})=\delta_{i j} K_{0}(\|\boldsymbol{x}\|)+x_{i} x_{j} K_{2}(\|\boldsymbol{x}\|) .
$$

Let $k_{\boldsymbol{x}}, \boldsymbol{x} \neq \mathbf{0}$, be the rotation with Euler angles $(0, \theta, \varphi)$, where $\theta$ and $\varphi$ are angular spherical coordinates of the point $\boldsymbol{x}$. We introduce the following notation: 


$$
\begin{aligned}
& M_{i j \ell m}^{1}(\boldsymbol{x})=\frac{1}{3} \delta_{i j} \delta_{\ell m}, \\
& M_{i j \ell m}^{2}(\boldsymbol{x})=\frac{1}{\sqrt{5}} \sum_{n=-2}^{2} g_{2[1,1]}^{n[i, j]} g_{2[1,1]}^{n[\ell, m]}, \\
& M_{i j \ell m}^{3}(\boldsymbol{x})=\frac{1}{\sqrt{6}}\left[\delta_{i j} \sum_{n=-2}^{2} g_{2[1,1]}^{n[\ell, m]} U_{n 0}^{2}\left(k_{\boldsymbol{x}}\right)+\delta_{\ell m} \sum_{n=-2}^{2} g_{2[1,1]}^{n[i, j]} U_{n 0}^{2}\left(k_{\boldsymbol{x}}\right)\right], \\
& M_{i j \ell m}^{4}(\boldsymbol{x})=\sum_{n=-2}^{2} \sum_{p=-2}^{2} g_{2[1,1]}^{n[i, j]} g_{2[1,1]}^{p[\ell, m]} \sum_{q=-2}^{2} g_{2[2,2]}^{q[n, p]} U_{q 0}^{2}\left(k_{\boldsymbol{x}}\right), \\
& M_{i j \ell m}^{5}(\boldsymbol{x})=\sum_{n=-2}^{2} \sum_{p=-2}^{2} g_{2[1,1]}^{n[i, j]} g_{2[1,1]}^{p[\ell, m]} \sum_{q=-4}^{4} g_{4[2,2]}^{q[n, p]} U_{q 0}^{4}\left(k_{\boldsymbol{x}}\right) .
\end{aligned}
$$

Theorem 3.2. Let $V$ be the space of all symmetric second-rank tensors over $\mathbb{R}^{3}$, let $\boldsymbol{T}(\boldsymbol{x})$ be a $V$-valued random field on $\mathbb{R}^{3}$ satisfying (1-1) with $U(k) \boldsymbol{T}=k \boldsymbol{T} k^{-1}$. Then $E_{i j}(\boldsymbol{x})=C \delta_{i j}, C \in \mathbb{R}$, and there exist five continuous functions $K_{1}, \ldots, K_{5}$ : $[0, \infty) \rightarrow \mathbb{R}$ with $K_{3}(0)=K_{4}(0)=K_{5}(0)=0$ such that

$$
B_{i j \ell m}(\boldsymbol{x})=\sum_{n=1}^{5} M_{i j \ell m}^{n}(\boldsymbol{x}) K_{n}(\|\boldsymbol{x}\|)
$$

A formula similar to (3-2) has been obtained by Lomakin [1964]. For any fixed $\boldsymbol{x} \in \mathbb{R}^{3}$, the tensor in the left-hand side of (3-2) is a symmetric linear operator acting in the space of symmetric tensors of the second rank. Following Boehler et al. [1994], denote the space of all such tensors by $T_{4}^{e}$. Under the action of $\mathrm{SO}(3)$, the space $T_{4}^{e}$ decomposes into the following direct sum:

$$
T_{4}^{e}=V^{0} \oplus V^{0} \oplus V^{2} \oplus V^{2} \oplus V^{4},
$$

where $V^{i}$ may be considered as the space of completely symmetric traceless tensors of the $i$-th rank linearly dependent on $x_{i}$. Using the general form of such a tensor given by invariant theory (see, for example, [Spencer 1971]), we obtain the result of [Lomakin 1964]:

$$
B_{i j \ell m}(\boldsymbol{x})=\sum_{n=1}^{5} L_{i j \ell m}^{n}(\boldsymbol{x}) K_{n}(\|\boldsymbol{x}\|),
$$

where (compare with [Boehler et al. 1994, Lemma, pp. 98-99]) 


$$
\begin{aligned}
L_{i j \ell m}^{1}(\boldsymbol{x}) & =\delta_{i j} \delta_{\ell m}, \\
L_{i j \ell m}^{2}(\boldsymbol{x}) & =\delta_{i \ell} \delta_{j m}+\delta_{i m} \delta_{j l}, \\
L_{i j \ell m}^{3}(\boldsymbol{x}) & =\frac{x_{j} x_{\ell}}{\|\boldsymbol{x}\|^{2}} \delta_{i m}+\frac{x_{i} x_{m}}{\|\boldsymbol{x}\|^{2}} \delta_{j \ell}+\frac{x_{i} x_{\ell}}{\|\boldsymbol{x}\|^{2}} \delta_{j m}+\frac{x_{j} x_{m}}{\|\boldsymbol{x}\|^{2}} \delta_{i \ell}, \\
L_{i j \ell m}^{4}(\boldsymbol{x}) & =\frac{x_{i} x_{j}}{\|\boldsymbol{x}\|^{2}} \delta_{\ell m}+\frac{x_{\ell} x_{m}}{\|\boldsymbol{x}\|^{2}} \delta_{i j}, \\
L_{i j \ell m}^{5}(\boldsymbol{x}) & =\frac{x_{i} x_{j} x_{\ell} x_{m}}{\|\boldsymbol{x}\|^{4}} .
\end{aligned}
$$

We prove that Theorem 3.2 is equivalent to the result of [Lomakin 1964]. Indeed, we have

$$
\begin{aligned}
M_{i j \ell m}^{1}(\boldsymbol{x}) & =\frac{1}{3} L_{i j \ell m}^{1}(\boldsymbol{x}), \\
M_{i j \ell m}^{2}(\boldsymbol{x}) & =-\frac{1}{3 \sqrt{5}} L_{i j \ell m}^{1}(\boldsymbol{x})+\frac{1}{2 \sqrt{5}} L_{i j \ell m}^{2}(\boldsymbol{x}), \\
M_{i j \ell m}^{3}(\boldsymbol{x}) & =-\frac{1}{3} L_{i j \ell m}^{1}(\boldsymbol{x})+\frac{1}{2} L_{i j \ell m}^{4}(\boldsymbol{x}), \\
M_{i j \ell m}^{4}(\boldsymbol{x}) & =\frac{2 \sqrt{2}}{3 \sqrt{7}} L_{i j \ell m}^{1}(\boldsymbol{x})-\frac{1}{\sqrt{14}} L_{i j \ell m}^{2}(\boldsymbol{x})+\frac{3}{2 \sqrt{14}} L_{i j \ell m}^{3}(\boldsymbol{x})-\frac{\sqrt{2}}{\sqrt{7}} L_{i j \ell m}^{4}(\boldsymbol{x}), \\
M_{i j \ell m}^{5}(\boldsymbol{x}) & =\frac{1}{2 \sqrt{70}} L_{i j \ell m}^{1}(\boldsymbol{x})+\frac{1}{2 \sqrt{70}} L_{i j \ell m}^{2}(\boldsymbol{x})-\frac{\sqrt{5}}{2 \sqrt{14}} L_{i j \ell m}^{3}(\boldsymbol{x}) \\
& -\frac{\sqrt{5}}{2 \sqrt{14}} L_{i j \ell m}^{4}(\boldsymbol{x})+\frac{\sqrt{35}}{2 \sqrt{2}} L_{i j \ell m}^{5}(\boldsymbol{x}) .
\end{aligned}
$$

It is easy to check that the transition matrix between Lomakin's functions (3-3) and the functions (3-1) is invertible. A proof of (3-4) may be found in the Appendix.

Given that $\boldsymbol{T}$ has diagonal and off-diagonal components, there are five special cases of $B_{i j \ell m}$ that shed light on the physical meaning of the $K_{n}$ :

(1) $\left.\mathrm{E}\left[T_{i j}(\mathbf{0}) T_{k l}(\boldsymbol{x})\right]\right|_{i=j=k=l}$; that is, auto-correlations of diagonal terms

$$
\mathrm{E}\left[T_{11}(\mathbf{0}) T_{11}(\boldsymbol{x})\right]=K_{1}+2 K_{2}+2 x_{1}^{2} K_{3}+4 x_{1}^{2} K_{4}+x_{1}^{4} K_{5}
$$

and then $\mathrm{E}\left[T_{22}(\mathbf{0}) T_{22}(\boldsymbol{x})\right]$ and $\mathrm{E}\left[T_{33}(\mathbf{0}) T_{33}(\boldsymbol{x})\right]$ by cyclic permutations $1 \rightarrow$ $2 \rightarrow 3$.

(2) $\left.\mathrm{E}\left[T_{i j}(\mathbf{0}) T_{k l}(\boldsymbol{x})\right]\right|_{i=j \neq k=l}$; that is, cross-correlations of diagonal terms

$$
\mathrm{E}\left[T_{11}(\mathbf{0}) T_{22}(\boldsymbol{x})\right]=K_{1}+\left(x_{2}^{2}+x_{1}^{2}\right) K_{3}+x_{2}^{2} x_{1}^{2} K_{5}
$$

and then $\mathrm{E}\left[T_{22}(\mathbf{0}) T_{33}(\boldsymbol{x})\right]$ and $\mathrm{E}\left[T_{33}(\mathbf{0}) T_{11}(\boldsymbol{x})\right]$ by cyclic permutations $1 \rightarrow$ $2 \rightarrow 3$. 
(3) $\left.\mathrm{E}\left[T_{i j}(\mathbf{0}) T_{k l}(\boldsymbol{x})\right]\right|_{i=k \neq j=l}$; that is, auto-correlations of off-diagonal terms

$$
\mathrm{E}\left[T_{12}(\mathbf{0}) T_{12}(\boldsymbol{x})\right]=K_{2}+\left(x_{1}^{2}+x_{2}^{2}\right) K_{4}+x_{1}^{2} x_{2}^{2} K_{5}
$$

and then $\mathrm{E}\left[T_{23}(\mathbf{0}) T_{23}(\boldsymbol{x})\right]$ and $\mathrm{E}\left[T_{31}(\mathbf{0}) T_{31}(\boldsymbol{x})\right]$ by cyclic permutations $1 \rightarrow$ $2 \rightarrow 3$.

(4) $\left.\mathrm{E}\left[T_{i j}(\mathbf{0}) T_{k l}(\boldsymbol{x})\right]\right|_{j \neq i=k \neq l \neq j}$; that is, cross-correlations of off-diagonal terms

$$
\mathrm{E}\left[T_{12}(\mathbf{0}) T_{13}(\boldsymbol{x})\right]=x_{2} x_{3} K_{4}+x_{1}^{2} x_{2} x_{3} K_{5}
$$

and then $\mathrm{E}\left[T_{13}(\mathbf{0}) T_{32}(\boldsymbol{x})\right]$ and $\mathrm{E}\left[T_{32}(\mathbf{0}) T_{12}(\boldsymbol{x})\right]$ by cyclic permutations $1 \rightarrow$ $2 \rightarrow 3$.

(5) $\left.\mathrm{E}\left[T_{i j}(\mathbf{0}) T_{k l}(\boldsymbol{x})\right]\right|_{i=j=k \neq l \neq j}$; that is, cross-correlations of diagonal terms with off-diagonal terms such as

$$
\mathrm{E}\left[T_{11}(\mathbf{0}) T_{12}(\boldsymbol{x})\right]=x_{1} x_{2}\left(K_{3}+2 K_{4}\right)+x_{1} x_{2}^{3} K_{5}
$$

and

$$
\mathrm{E}\left[T_{12}(\mathbf{0}) T_{13}(\boldsymbol{x})\right]=x_{2} x_{3} K_{3}+x_{1}^{2} x_{2} x_{3} K_{5}
$$

and the others by cyclic permutations $1 \rightarrow 2 \rightarrow 3$.

In principle, we can determine these five correlations for a specific physical situation. For example, when $\boldsymbol{T}$ is the antiplane elasticity tensor for a given resolution (or mesoscale) [Ostoja-Starzewski 2008], we can use micromechanics or experiments and then determine the best fits of the $K_{n}(n=1, \ldots, 5)$ coefficients.

\section{Concluding remarks}

Remark 4.1. On the one hand, Lomakin's functions (3-3) are simpler than functions (3-1). On the other hand, the restrictions of the functions (3-1) to the unit sphere $S^{2} \subset \mathbb{R}^{3}$ are orthogonal in the space of the square-integrable functions on $S^{2}$. Using this property, in a forthcoming paper we will obtain spectral expansions of tensor-valued homogeneous and isotropic random fields similar to those of Yaglom [1957].

Remark 4.2. The spherical harmonics $Y_{\ell m}(\theta, \varphi)$ are proportional to the Wigner $D$-functions by [Varshalovich et al. 1975, Equation (37), §5.2]:

$$
Y_{\ell m}(\theta, \varphi)=(-1)^{m} \sqrt{\frac{2 \ell+1}{4 \pi}} D_{-m 0}^{\ell}(0, \theta, \varphi) .
$$

The matrix entries $U_{\ell 0}^{i}\left(k_{x}\right)$ are proportional to the zonal, sectorial, and tesseral harmonics defined by [Varshalovich et al. 1975, Equation (14), §5.1]. 
Remark 4.3. The matrix entries $U_{j 0}^{i}\left(k_{x}\right)$, the Godunov-Gordienko coefficients, and (3-4) were calculated and proved by hand. Afterwards, they were checked using MATLAB and Symbolic Math Toolbox ${ }^{\mathrm{TM}}$ [Mathworks 2013]. The problem of an algebraic proof of the second and fourth equations in (3-4) remains open.

\section{Appendix: Proofs}

Proof of Lemma 2.2. The two-point correlation function $B(\boldsymbol{x})$ is a linear operator in $V$. It is known that the space of linear operators in $V$ is isomorphic to the tensor product $V^{*} \otimes V$, where $V^{*}$ is the set of all $\mathbb{R}$-linear maps $\boldsymbol{v}^{*}: V \rightarrow \mathbb{R}$. We need to prove that for any $S \in V^{*} \otimes V$ the following equality holds true:

$$
U(k) \mathrm{SU}^{-1}(k)=(U \otimes U)(k) S .
$$

Note that the set of tensors satisfying the above equality form a linear space. Therefore, it is enough to prove this equality for tensors of the form $\boldsymbol{v}^{*} \otimes \boldsymbol{v}$, where $\boldsymbol{v} \in V$ and $\boldsymbol{v}^{*} \in V^{*}$. The linear operator $\boldsymbol{v}^{*} \otimes \boldsymbol{v}$ acts on $V$ by

$$
\left(\boldsymbol{v}^{*} \otimes \boldsymbol{v}\right) \boldsymbol{w}=\boldsymbol{v}^{*}(\boldsymbol{w}) \boldsymbol{v}, \quad \boldsymbol{w} \in V .
$$

For this operator,

$$
\begin{aligned}
\left(U(k) \boldsymbol{v}^{*} \otimes \boldsymbol{v} U^{-1}(k)\right) \boldsymbol{w} & =U(k) \boldsymbol{v}^{*}\left(U^{-1}(k) \boldsymbol{w}\right) \boldsymbol{v}=\left(U(k) \boldsymbol{v}^{*}\right)(\boldsymbol{w}) U(k) \boldsymbol{v} \\
& =\left(U(k) \boldsymbol{v}^{*}\right) \otimes(U(k) \boldsymbol{v}) \boldsymbol{w}=(U \otimes U)(k)\left(\boldsymbol{v}^{*} \otimes \boldsymbol{v}\right) \boldsymbol{w} .
\end{aligned}
$$

By linearity, this equality follows for any $S \in V^{*} \otimes V$.

Proof of Theorem 3.1. The first equation in (1-1) may be written in the Gordienko basis as

$$
U^{1}(k) \boldsymbol{E}=\boldsymbol{E} .
$$

The representation $U^{1}$ is irreducible. By Lemma 2.1, $\boldsymbol{E}=\mathbf{0}$.

We cannot apply Lemma 2.1 to (2-11), because the representation $U^{1} \otimes U^{1}$ acting in $V^{1} \otimes V^{1}$ is reducible.

By definition, a tensor field $B(x)$ is a function on $\mathbb{R}^{3}$ with values in the linear space of real-valued bilinear forms on $V^{1} \times V^{1}$. The component $B_{i j}(x)$ is the value of the bilinear form $B(\boldsymbol{x})$ at the point $\left(\boldsymbol{h}_{i}^{1}, \boldsymbol{h}_{j}^{1}\right) \in V^{1} \times V^{1}$ :

$$
B_{i j}(\boldsymbol{x})=B\left(\boldsymbol{x} ; \boldsymbol{h}_{i}^{1}, \boldsymbol{h}_{j}^{1}\right) .
$$

The last equality may be rewritten as

$$
B_{i j}(\boldsymbol{x})=B\left(\boldsymbol{x} ; \boldsymbol{h}_{i}^{1} \otimes \boldsymbol{h}_{j}^{1}\right),
$$

where the right-hand side is the value of the linear form $B(\boldsymbol{x})$ at the point $\boldsymbol{h}_{i}^{1} \otimes \boldsymbol{h}_{j}^{1} \in$ $V^{1} \otimes V^{1}$. In other words, tensor products simplify multilinear forms to linear ones. 
Using (2-9) with $m=p=1, n=1$, and $q=j$, we obtain

$$
\begin{aligned}
B_{i j}(\boldsymbol{x}) & =B\left(\boldsymbol{x} ; \boldsymbol{h}_{i}^{1} \otimes \boldsymbol{h}_{j}^{1}\right)=B\left(\boldsymbol{x} ; \sum_{m=0}^{2} \sum_{\ell=-m}^{m} g_{m[1,1]}^{\ell[i, j]} \boldsymbol{h}_{\ell}^{m}\right) \\
& =\sum_{m=0}^{2} \sum_{\ell=-m}^{m} g_{m[1,1]}^{\ell[i, j]} B\left(\boldsymbol{x} ; \boldsymbol{h}_{\ell}^{m}\right) .
\end{aligned}
$$

Let $\boldsymbol{B}_{m}(\boldsymbol{x}) \in V^{m}$ be the vector field with components $B\left(\boldsymbol{x} ; \boldsymbol{h}_{\ell}^{m}\right),-m \leq \ell \leq m$. For this field, we have

$$
\boldsymbol{B}_{m}(k \boldsymbol{x})=U^{m}(k) \boldsymbol{B}_{m}(\boldsymbol{x}), \quad k \in \mathrm{SO}(3) .
$$

By Lemma 2.1, $\boldsymbol{B}_{m}(\mathbf{0})=\mathbf{0}$ for $m \geq 1$, while $\boldsymbol{B}_{0}(\mathbf{0})$ may take any real value.

Let $\mathrm{SO}(2)$ be the subgroup of rotations around $x_{0}$ axis. The restriction of the representation $U^{m}$ to $\mathrm{SO}(2)$ is the direct sum of the trivial representation $W^{0}$ of $\mathrm{SO}(2)$ acting in the one-dimensional space spanned by the vector $\boldsymbol{h}_{0}^{m}$ and of the irreducible representations

$$
W^{\ell}(\varphi)=\left(\begin{array}{cc}
\cos (\ell \varphi) & \sin (\ell \varphi) \\
-\sin (\ell \varphi) & \cos (\ell \varphi)
\end{array}\right), \quad \varphi \in \operatorname{SO}(2),
$$

acting in the two-dimensional spaces spanned by the vectors $\boldsymbol{h}_{\ell}^{m}$ and $\boldsymbol{h}_{-\ell}^{m}, 1 \leq \ell \leq m$. If $\boldsymbol{x}=(0,\|\boldsymbol{x}\|, 0)^{\top} \neq \mathbf{0}$, then $k \boldsymbol{x}=\boldsymbol{x}, k \in \mathrm{SO}(2)$, and

$$
\left(B\left(\boldsymbol{x} ; \boldsymbol{h}_{-\ell}^{m}\right), B\left(\boldsymbol{x} ; \boldsymbol{e}_{\ell}^{m}\right)\right)^{\top}=W^{\ell}(\varphi)\left(B\left(\boldsymbol{x} ; \boldsymbol{h}_{-\ell}^{m}\right), B\left(\boldsymbol{x} ; \boldsymbol{h}_{\ell}^{m}\right)\right)^{\top}, \quad 1 \leq \ell \leq m .
$$

By Lemma 2.1, $B\left(\boldsymbol{x} ; \boldsymbol{h}_{-\ell}^{m}\right)=B\left(\boldsymbol{x} ; \boldsymbol{h}_{\ell}^{m}\right)=0$, if $1 \leq \ell \leq m$, while $B\left(\boldsymbol{x} ; \boldsymbol{h}_{0}^{m}\right)$ is an arbitrary continuous real-valued function with $B\left(\mathbf{0} ; \boldsymbol{h}_{0}^{m}\right)=0$ for $m \geq 1$. In what follows, we denote this function by $B_{m}(\|x\|)$.

By (A.2),

$$
B\left(\boldsymbol{x} ; \boldsymbol{h}_{\ell}^{m}\right)=U_{\ell 0}^{m}\left(k_{\boldsymbol{x}}\right) B_{m}(\|\mathbf{x}\|) .
$$

Substitute (A.3) into (A.1). We obtain

$$
B_{i j}(\boldsymbol{x})=\sum_{m=0}^{2} \sum_{\ell=-m}^{m} g_{m[1,1]}^{\ell[i, j]} U_{\ell 0}^{m}\left(k_{\boldsymbol{x}}\right) B_{m}(\|\boldsymbol{x}\|) .
$$

In other words, $B_{i j}(\boldsymbol{x})$ is the sum of three matrix-valued functions

$$
B_{i j}(\boldsymbol{x})=\sum_{m=0}^{2} B_{i j}^{m}(\boldsymbol{x}),
$$

with

$$
B_{i j}^{m}(\boldsymbol{x})=\sum_{\ell=-m}^{m} g_{m[1,1]}^{\ell[i, j]} U_{\ell 0}^{m}\left(k_{\boldsymbol{x}}\right) B_{m}(\|\boldsymbol{x}\|) .
$$


Let $m=0$. Then

$$
U_{00}^{0}\left(k_{x}\right)=1,
$$

the matrix entry of the $1 \times 1$ identity matrix. To calculate the Godunov-Gordienko coefficients $g_{0[1,1]}^{0[i, j]}$, use the following property [Godunov and Gordienko 2004]:

$$
g_{N\left[N_{1}, N_{2}\right]}^{n\left[n_{1}, n_{2}\right]}=(-1)^{N+N_{1}+N_{2}} \sqrt{\frac{2 N+1}{2 N_{1}+1}} g_{N_{1}\left[N, N_{2}\right]}^{n_{1}\left[n, n_{2}\right]},
$$

with $N=n=0, N_{1}=N_{2}=1, n_{1}=i$, and $n_{2}=j$. We have

$$
g_{0[1,1]}^{0[i, j]}=\sqrt{1 / 3} g_{1[0,1]}^{i[0, j]} \text {. }
$$

The coefficients in the right-hand side can be calculated using (2-9):

$$
\boldsymbol{h}_{0}^{0} \otimes \boldsymbol{h}_{j}^{1}=\sum_{i=-1}^{1} g_{1[0,1]}^{i[0, j]} \boldsymbol{h}_{i}^{1}
$$

The left-hand side is clearly equal to $\boldsymbol{h}_{j}^{1}$. Therefore, $g_{1[0,1]}^{i[0, j]}=\delta_{i j}$,

$$
g_{0[1,1]}^{0[i, j]}=\sqrt{1 / 3} \delta_{i j}
$$

and the first matrix is

$$
B_{i j}^{0}(\boldsymbol{x})=\sqrt{1 / 3} \delta_{i j} B_{0}(\|\boldsymbol{x}\|) .
$$

Let $m=1$. Using (2-5)-(2-7), we obtain

$$
\left(\begin{array}{c}
U_{-10}^{1}(\varphi, \theta) \\
U_{00}^{1}(\varphi, \theta) \\
U_{10}^{1}(\varphi, \theta)
\end{array}\right)=\left(\begin{array}{ccc}
\cos \varphi & 0 & \sin \varphi \\
0 & 1 & 0 \\
-\sin \varphi & 0 & \cos \varphi
\end{array}\right)\left(\begin{array}{c}
0 \\
\cos \theta \\
\sin \theta
\end{array}\right)=\left(\begin{array}{c}
\sin \varphi \sin \theta \\
\cos \theta \\
\cos \varphi \sin \theta
\end{array}\right),
$$

or

$$
U_{\ell 0}^{1}\left(k_{x}\right)=\frac{x_{\ell}}{r} .
$$

The Godunov-Gordienko coefficients $g_{1[1,1]}^{\ell[i, j]}$ are calculated by [Godunov and Gordienko 2004, Formulae (1.26)-(1.29)]. The nonzero coefficients are

$$
\begin{aligned}
& g_{1[1,1]}^{-1[1,0]}=g_{1[1,1]}^{0[-1,1]}=g_{1[1,1]}^{1[0,-1]}=\sqrt{1 / 2}, \\
& g_{1[1,1]}^{-1[0,1]}=g_{1[1,1]}^{0[1,-1]}=g_{1[1,1]}^{1[-1,0]}=-\sqrt{1 / 2} .
\end{aligned}
$$

The second matrix becomes

$$
\frac{1}{r \sqrt{2}}\left(\begin{array}{ccc}
0 & -x_{1} & x_{0} \\
x_{1} & 0 & -x_{-1} \\
-x_{0} & x_{-1} & 0
\end{array}\right) B_{1}(\|\boldsymbol{x}\|) .
$$


This matrix is not symmetric but skew-symmetric. The matrix $B_{i j}(\boldsymbol{x})$ is symmetric if and only if $B_{1}(\|x\|)=0$.

The matrix entries $U_{\ell 0}^{2}\left(k_{x}\right)$ can be calculated using (2-5)-(2-7) as

$$
\begin{aligned}
U_{-20}^{2}\left(k_{x}\right) & =-\frac{\sqrt{3} x_{-1} x_{1}}{r^{2}}, & U_{-10}^{2}\left(k_{x}\right) & =\frac{\sqrt{3} x_{-1} x_{0}}{r^{2}}, \quad U_{00}^{2}\left(k_{x}\right)=\frac{3 x_{0}^{2}}{2 r^{2}}-\frac{1}{2}, \\
U_{10}^{2}\left(k_{x}\right) & =\frac{\sqrt{3} x_{0} x_{1}}{r^{2}}, & U_{20}^{2}\left(k_{x}\right) & =\frac{\sqrt{3}\left(x_{-1}^{2}-x_{1}^{2}\right)}{2 r^{2}} .
\end{aligned}
$$

To calculate the Godunov-Gordienko coefficients $g_{2[1,1]}^{\ell[i, j]}$, use (A.5) with $N=2$, $N_{1}=N_{2}=1, n=\ell, n_{1}=i$, and $n_{2}=j$. We have

$$
g_{2[1,1]}^{\ell[i, j]}=\sqrt{5 / 3} g_{1[2,1]}^{i[\ell, j]} .
$$

The coefficients $g_{1[2,1]}^{i[\ell, j]}$ are calculated by [Godunov and Gordienko 2004, Formulae (1.30)-(1.34)]. The nonzero Godunov-Gordienko coefficients are

$$
\begin{aligned}
g_{2[1,1]}^{-2[-1,1]} & =g_{2[1,1]}^{-2[1,-1]}=g_{2[1,1]}^{2[1,1]}=-\sqrt{1 / 2}, \\
g_{2[1,1]}^{0[-1,-1]} & =g_{2[1,1]}^{0[1,1]}=-\sqrt{1 / 6}, \\
g_{2[1,1]}^{-1[-1,0]} & =g_{2[1,1]}^{-1[0,-1]}=g_{2[1,1]}^{1[0,1]}=g_{2[1,1]}^{1[1,0]}=g_{2[1,1]}^{2[-1,-1]}=\sqrt{1 / 2}, \\
g_{2[1,1]}^{0[0,0]} & =\sqrt{2 / 3} .
\end{aligned}
$$

In (2-10) put $k=j=0, m=p=1, n=i$, and $q=j$. We have

$$
U_{i 0}^{1}(k) U_{j 0}^{1}(k)=\sum_{s=0}^{2} \sum_{t=-s}^{s} \sum_{\ell=-s}^{s} g_{s[1,1]}^{t[i, j]} U_{t \ell}^{s}(k) g_{s[1,1]}^{\ell[0,0]} .
$$

Using the above-calculated values of the matrix entries and Godunov-Gordienko coefficients, we may rewrite this formula as

$$
\frac{x_{i} x_{j}}{r^{2}}=\frac{1}{3} \delta_{i j}+\sqrt{\frac{2}{3}} \sum_{t=-2}^{2} g_{2[1,1]}^{t[i, j]} U_{t 0}^{2}\left(k_{x}\right)
$$

or

$$
\sum_{t=-2}^{2} g_{2[1,1]}^{t[i, j]} U_{t 0}^{2}\left(k_{x}\right)=\frac{\sqrt{3}}{\sqrt{2} r^{2}}\left(x_{j} x_{j}-\frac{r^{2}}{3} \delta_{i j}\right) .
$$

We introduce the following notation:

$$
K_{0}(\|x\|)=\frac{1}{\sqrt{3}} B_{0}(\|x\|)-\frac{1}{\sqrt{6}} B_{2}(\|x\|), \quad K_{2}(\|x\|)=\frac{\sqrt{3}}{\sqrt{2} r^{2}} B_{2}(\|x\|) .
$$

The theorem is proved. Note that the space $V^{0}$ consists of tensors proportional to the Kronecker delta, $V^{1}$ consists of skew-symmetric tensors, and $V^{2}$ consists of symmetric traceless tensors. 
Proof of Theorem 3.2. Use Lemma 2.2 to write the representation $U$ as $U=$ $U^{1} \otimes U^{1}$. The first equation in (1-1) takes the form

$$
E(k \boldsymbol{x})=\left(U^{1} \otimes U^{1}\right)(k) E(\boldsymbol{x}) .
$$

or

$$
E=\left(U^{1} \otimes U^{1}\right)(k) E, \quad k \in \mathrm{SO}(3) .
$$

In other words, $E$ lies in the subspace of $V$ where the trivial representation is realized, or

$$
\mathrm{E}\left[T_{i j}(\boldsymbol{x})\right]=C \delta_{i j}, \quad C \in \mathbb{R} .
$$

The second equation in (1-1) takes the form

$$
B(k \boldsymbol{x})=\left(U^{1} \otimes U^{1} \otimes U^{1} \otimes U^{1}\right)(k) B(\boldsymbol{x}) .
$$

We write $B(\boldsymbol{x})$ as a bilinear form on pairs of tensors, $\left(V^{1} \otimes V^{1}\right) \times\left(V^{1} \otimes V^{1}\right)$ :

$$
B^{i j k \ell}(\boldsymbol{x})=B\left(\boldsymbol{x} ; \boldsymbol{h}_{i}^{1} \otimes \boldsymbol{h}_{j}^{1}, \boldsymbol{h}_{\ell}^{1} \otimes \boldsymbol{h}_{m}^{1}\right) .
$$

Note that the tensor field $B^{i j \ell m}(\boldsymbol{x})$ is symmetric in the following sense:

$$
B^{\ell m i j}(\boldsymbol{x})=B^{i j \ell m}(\boldsymbol{x}) .
$$

Recall that $V^{1} \otimes V^{1}=V^{0} \oplus V^{1} \oplus V^{2}$. Rewrite $B(x)$ as a bilinear form on $\left(V^{0} \oplus V^{1} \oplus V^{2}\right) \times\left(V^{0} \oplus V^{1} \oplus V^{2}\right)$ :

$$
B^{i j \ell m}(\boldsymbol{x})=B\left(\boldsymbol{x} ; \sum_{n=0}^{2} \sum_{p=-n}^{n} g_{n[1,1]}^{p[i, j]} \boldsymbol{h}_{p}^{n}, \sum_{q=0}^{2} \sum_{r=-q}^{q} g_{q[1,1]}^{r[\ell, m]} \boldsymbol{h}_{r}^{q}\right) .
$$

In fact, $B(\boldsymbol{x})$ is a bilinear form on pairs of symmetric tensors, which is to say, on $\left(V^{0} \oplus V^{2}\right) \times\left(V^{0} \oplus V^{2}\right)$ :

$$
\begin{aligned}
B^{i j \ell m}(\boldsymbol{x}) & =B\left(\boldsymbol{x} ; \sum_{n \in\{0,2\}} \sum_{p=-n}^{n} g_{n[1,1]}^{p[i, j]} \boldsymbol{h}_{p}^{n}, \sum_{q \in\{0,2\}} \sum_{r=-q}^{q} g_{q[1,1]}^{r[\ell, m]} \boldsymbol{h}_{r}^{q}\right) \\
& =\sum_{(n, q) \in\{0,2\} \times\{0,2\}} \sum_{p=-n}^{n} \sum_{r=-q}^{q} g_{n[1,1]}^{p[i, j]} g_{q[1,1]}^{r[\ell, m]} B\left(\boldsymbol{x} ; \boldsymbol{h}_{p}^{n}, \boldsymbol{h}_{r}^{q}\right) \\
& =\sum_{(n, q) \in\{0,2\} \times\{0,2\}} \sum_{p=-n}^{n} \sum_{r=-q}^{q} g_{n[1,1]}^{p[i, j]} g_{q[1,1]}^{r[\ell, m]} B\left(\boldsymbol{x} ; \boldsymbol{h}_{p}^{n} \otimes \boldsymbol{h}_{r}^{q}\right) \\
& =\sum_{(n, q) \in\{0,2\} \times\{0,2\}} \sum_{p=-n}^{n} \sum_{r=-q}^{q} g_{n[1,1]}^{p[i, j]} g_{q[1,1]}^{r[\ell, m]} B\left(\boldsymbol{x} ; \sum_{s=|n-q|}^{n+q} \sum_{t=-s}^{s} g_{s[m, p]}^{t[n, q]} \boldsymbol{h}_{t}^{s}\right) .
\end{aligned}
$$

The possible values for $s$ are $0 \leq s \leq 4$. Let $A_{s}$ be the set of all pairs $(n, q) \in$ $\{0,2\} \times\{0,2\}$ such that $V^{s} \subseteq V^{n} \otimes V^{q}$. We have 
$A_{0}=\{(0,0),(2,2)\}, \quad A_{1}=A_{3}=A_{4}=\{(2,2)\}, \quad A_{2}=\{(0,2),(2,0),(2,2)\}$,

and

$$
B^{i j \ell m}(\boldsymbol{x})=\sum_{s=0}^{4} B_{s}^{i j \ell m}(\boldsymbol{x})
$$

where

$$
B_{s}^{i j \ell m}(\boldsymbol{x})=\sum_{(n, q) \in A_{s}} \sum_{p=-n}^{n} \sum_{r=-q}^{q} g_{n[1,1]}^{p[i, j]} g_{q[1,1]}^{r[\ell, m]} \sum_{t=-s}^{s} g_{s[m, p]}^{t[n, q]} B\left(\boldsymbol{x} ; \boldsymbol{h}_{t}^{s}(n, q)\right),
$$

and where the pairs $(n, q) \in A_{s}$ enumerate copies of the vector $\boldsymbol{h}_{t}^{s}$. By (A.3),

$$
B\left(\boldsymbol{x} ; \boldsymbol{h}_{t}^{s}(n, q)\right)=U_{t 0}^{s}\left(k_{x}\right) B_{s(n, q)}(\|\boldsymbol{x}\|),
$$

where $B_{s(n, q)}(\|\boldsymbol{x}\|)$ are continuous real-valued functions with $B_{s(n, q)}(0)=0$ for $s \geq 1$. We obtain

$$
B_{s}^{i j \ell m}(\boldsymbol{x})=\sum_{(n, q) \in A_{s}} C_{n q s}^{i j \ell m}(\boldsymbol{x})
$$

where

$$
C_{n q s}^{i j \ell m}(\boldsymbol{x})=\sum_{p=-n}^{n} \sum_{r=-q}^{q} g_{n[1,1]}^{p[i, j]} g_{q[1,1]}^{r[\ell, m]} \sum_{t=-s}^{s} g_{s[n, q]}^{t[p, r]} U_{t 0}^{s}\left(k_{\boldsymbol{x}}\right) B_{s(n, q)}(\|\boldsymbol{x}\|) .
$$

We calculate the functions $C_{n q s}^{i j \ell m}(\boldsymbol{x})$.

Let $(n, q)=(0,0) \in A_{0}$. The corresponding term is

$$
C_{000}^{i j \ell m}(\boldsymbol{x})=\frac{1}{3} \delta_{i j} \delta_{\ell m} B_{0(0,0)}(\|\boldsymbol{x}\|)=M_{i j \ell m}^{1}(\boldsymbol{x}) K_{1}(\|\boldsymbol{x}\|),
$$

by (A.4) and (A.6) with $K_{1}(\|\boldsymbol{x}\|)=B_{0(0,0)}(\|\boldsymbol{x}\|)$.

Let $(n, q)=(2,2) \in A_{0}$. The corresponding term is

$$
C_{220}^{i j \ell m}(\boldsymbol{x})=\sum_{r=-2}^{2} \sum_{s=-2}^{2} g_{2[1,1]}^{r[i, j]} g_{2[1,1]}^{s[\ell, m]} g_{0[2,2]}^{0[r, s]} U_{00}^{0}\left(k_{\boldsymbol{x}}\right) B_{0(2,2)}(\|\boldsymbol{x}\|) .
$$

The Godunov-Gordienko coefficients $g_{2[1,1]}^{r[i, j]}$ and $g_{2[1,1]}^{s[\ell, m]}$ are calculated by (A.6), while the coefficients $g_{0[2,2]}^{0[n, q]}$ are calculated using (A.5) with $n=N=0, n_{1}=r$, $n_{2}=s, N_{1}=N_{2}=2$ and (2-9):

$$
g_{0[2,2]}^{0[r, s]}=\sqrt{1 / 5} g_{2[0,2]}^{r[0, s]}=\sqrt{1 / 5} \delta_{r s} .
$$

Therefore, we obtain

$$
C_{220}^{i j \ell m}(\boldsymbol{x})=\sqrt{1 / 5} \sum_{r=-2}^{2} g_{2[1,1]}^{r[i, j]} g_{2[1,1]}^{r[\ell, m]} B_{0(2,2)}(\|x\|)=M_{i j \ell m}^{2}(\boldsymbol{x}) K_{2}(\|\boldsymbol{x}\|)
$$

with $K_{2}(\|\boldsymbol{x}\|)=B_{0(2,2)}(\|\boldsymbol{x}\|)$. 
Let $(n, q)=(2,2) \in A_{1}$. The corresponding term is

$$
C_{221}^{i j \ell m}(\boldsymbol{x})=\sum_{p=-2}^{2} \sum_{s=-2}^{2} g_{2[1,1]}^{p[i, j]} g_{2[1,1]}^{r[\ell, m]} \sum_{s=-1}^{1} g_{1[2,2]}^{s[p, r]} U_{s 0}^{1}\left(k_{\boldsymbol{x}}\right) B_{1(2,2)}(\|\boldsymbol{x}\|) .
$$

The Godunov-Gordienko coefficients $g_{1[2,2]}^{s[p, r]}$ are calculated by [Godunov and Gordienko 2004, Formulae (1.26)-(1.29)]. The nonzero elements of the GodunovGordienko matrix $G_{1[2,2]}^{-1}$ are

$$
\begin{array}{ll}
g_{1[2,2]}^{-1[0,1]}=-\sqrt{3 / 10}, & g_{1[2,2]}^{-1[-2,-1]}=g_{1[2,2]}^{-1[2,1]}=-\sqrt{1 / 10}, \\
g_{1[2,2]}^{-1[1,0]}=\sqrt{3 / 10}, & g_{1[2,2]}^{-1[-1,-2]}=g_{1[2,2]}^{-1[1,2]}=\sqrt{1 / 10},
\end{array}
$$

and those of the matrix $G_{1[2,2]}^{0}$ are

$$
\begin{array}{ll}
g_{1[2,2]}^{0[2,-2]}=-\sqrt{2 / 5}, & g_{1[2,2]}^{0[1,-1]}=-\sqrt{1 / 10}, \\
g_{1[2,2]}^{0[-2,2]}=\sqrt{2 / 5}, & g_{1[2,2]}^{0[-1,1]}=\sqrt{1 / 10} .
\end{array}
$$

Finally, the nonzero elements of the Godunov-Gordienko matrix $G_{1[2,2]}^{-1}$ are

$$
\begin{array}{ll}
g_{1[2,2]}^{1[0,-1]}=\sqrt{3 / 10}, & g_{1[2,2]}^{1[1,-2]}=g_{1[2,2]}^{1[2,-1]}=-\sqrt{1 / 10}, \\
g_{1[2,2]}^{1[-1,0]}=-\sqrt{3 / 10}, & g_{1[2,2]}^{1[-2,1]}=g_{1[2,2]}^{1[-1,2]}=\sqrt{1 / 10} .
\end{array}
$$

We see that the Godunov-Gordienko matrices $g_{1[2,2]}^{s},-1 \leq s \leq 1$, are skewsymmetric. We have $B_{1(2,2)}(\|\boldsymbol{x}\|)=0$ by the same reasons as $B_{1}(\|\boldsymbol{x}\|)=0$ in the proof of Theorem 3.1.

Let $(n, q)=(0,2) \in A_{2}$. The corresponding term is

$$
C_{022}^{i j \ell m}(\boldsymbol{x})=g_{0[1,1]}^{0[i, j]} \sum_{p=-2}^{2} g_{2[1,1]}^{p[\ell, m]} \sum_{s=-2}^{2} g_{2[0,2]}^{s[0, p]} U_{s 0}^{2}\left(k_{\boldsymbol{x}}\right) B_{2(0,2)}(\|\boldsymbol{x}\|) .
$$

Using (A.6), we obtain

$$
C_{022}^{i j \ell m}(\boldsymbol{x})=\sqrt{1 / 3} \delta_{i j} \sum_{p=-2}^{2} g_{2[1,1]}^{p[\ell, m]} \sum_{s=-2}^{2} g_{2[0,2]}^{s[0, r]} U_{s 0}^{2}\left(k_{\boldsymbol{x}}\right) B_{2(0,2)}(\|\boldsymbol{x}\|) .
$$

Clearly $g_{2[0,2]}^{s[0, p]}=\delta_{p s}$. Therefore,

$$
C_{022}^{i j \ell m}(\boldsymbol{x})=\sqrt{1 / 3} \delta_{i j} \sum_{p=-2}^{2} g_{2[1,1]}^{p[\ell, m]} U_{p 0}^{2}\left(k_{x}\right) B_{2(0,2)}(\|\boldsymbol{x}\|) .
$$

Let $(n, q)=(2,0) \in A_{2}$. The corresponding term is

$$
C_{202}^{i j \ell m}(\boldsymbol{x})=g_{0[1,1]}^{0[\ell, m]} \sum_{p=-2}^{2} g_{2[1,1]}^{p[i, j]} \sum_{s=-2}^{2} g_{2[2,0]}^{t[p, 0]} U_{s 0}^{2}\left(k_{\boldsymbol{x}}\right) B_{2(2,0)}(\|\boldsymbol{x}\|) .
$$


Using (A.6), we obtain

$$
C_{202}^{i j \ell m}(\boldsymbol{x})=\sqrt{1 / 3} \delta_{\ell m} \sum_{p=-2}^{2} g_{2[1,1]}^{p[i, j]} \sum_{s=-2}^{2} g_{2[2,0]}^{t[p, 0]} U_{s 0}^{2}\left(k_{x}\right) B_{2(2,0)}(\|\boldsymbol{x}\|) .
$$

Clearly $g_{2[2,0]}^{s[p, 0]}=\delta_{p s}$. Therefore,

$$
C_{202}^{i j \ell m}(\boldsymbol{x})=\sqrt{1 / 3} \delta_{\ell m} \sum_{p=-2}^{2} g_{2[1,1]}^{p[i, j]} U_{p 0}^{2}\left(k_{\boldsymbol{x}}\right) B_{2(2,0)}(\|\boldsymbol{x}\|) .
$$

We see that the tensor field $B^{i j \ell m}(x)$ is symmetric if and only if $B_{2(0,2)}(\|x\|)=$ $B_{2(2,0)}(\|\boldsymbol{x}\|)$. Denote their common value by $K_{3}(\|\boldsymbol{x}\|)$ and put

$$
C_{2}^{i j \ell m}(\boldsymbol{x})=\frac{1}{\sqrt{2}}\left(C_{022}^{i j \ell m}(\boldsymbol{x})+C_{202}^{i j \ell m}(\boldsymbol{x})\right) .
$$

We obtain

$$
C_{2}^{i j \ell m}(\boldsymbol{x})=M_{i j \ell m}^{3}(\boldsymbol{x}) K_{3}(\|\boldsymbol{x}\|) .
$$

Let $(n, q)=(2,2) \in A_{2}$. The corresponding term is

$$
\begin{aligned}
C_{222}^{i j \ell m}(\boldsymbol{x}) & =\sum_{p=-2}^{2} \sum_{r=-2}^{2} g_{2[1,1]}^{p[i, j]} g_{2[1,1]}^{r[\ell, m]} \sum_{s=-2}^{2} g_{2[2,2]}^{s[p, r]} U_{s 0}^{2}\left(k_{x}\right) B_{2(2,2)}(\|\boldsymbol{x}\|) \\
& =M_{i j \ell m}^{4}(\boldsymbol{x}) K_{4}(\|\boldsymbol{x}\|),
\end{aligned}
$$

with $K_{4}(\|\boldsymbol{x}\|)=B_{2(2,2)}(\|\boldsymbol{x}\|)$.

To calculate Godunov-Gordienko coefficients $g_{2[2,2]}^{s[p, r]}$, we use generating function from [Godunov and Gordienko 2004, Formula (2.6)]. The nonzero coefficients are:

$$
\begin{aligned}
g_{2[2,2]}^{-2[0,-2]} & =g_{2[2,2]}^{-2[-2,0]}=g_{2[2,2]}^{0[-2,-2]}=g_{2[2,2]}^{0[2,2]}=g_{2[2,2]}^{2[2,0]}=g_{2[2,2]}^{2[0,2]}=-\sqrt{2 / 7}, \\
g_{2[2,2]}^{-2[1,-1]} & =g_{2[2,2]}^{-2[-1,1]}=g_{2[2,2]}^{-1[1,-2]}=g_{2[2,2]}^{-1[-2,1]}=g_{2[2,2]}^{1[-1,-2]}=g_{2[2,2]}^{1[-2,-1]}=g_{2[2,2]}^{1[2,1]} \\
& =g_{2[2,2]}^{1[1,2]}=g_{2[2,2]}^{2[1,1]}=-\sqrt{3 / 14}, \\
g_{2[2,2]}^{-1[0,-1]} & =g_{2[2,2]}^{-1[-1,0]}=g_{2[2,2]}^{0[-1,-1]}=g_{2[2,2]}^{0[1,1]}=g_{2[2,2]}^{1[0,1]}=g_{2[2,2]}^{1[1,0]}=\sqrt{1 / 14}, \\
g_{2[2,2]}^{-1[2,-1]} & =g_{2[2,2]}^{-1[-1,2]}=g_{2[2,2]}^{2[-1,-1]}=\sqrt{3 / 14}, \\
g_{2[2,2]}^{0[0,0]} & =\sqrt{2 / 7} .
\end{aligned}
$$

Let $(n, q)=(2,2) \in A_{3}$. The corresponding term is

$$
C_{223}^{i j \ell m}(\boldsymbol{x})=\sum_{r=-2}^{2} \sum_{s=-2}^{2} g_{2[1,1]}^{r[i, j]} g_{2[1,1]}^{s[\ell, m]} \sum_{t=-3}^{3} g_{3[2,2]}^{t[r, s]} U_{t 0}^{3}\left(k_{x}\right) B_{3(2,2)}(\|\boldsymbol{x}\|) .
$$


The nonzero elements of the Godunov-Gordienko matrix $g_{3[2,2]}^{-3}$ are

$$
g_{3[2,2]}^{-3[-1,-2]}=g_{3[2,2]}^{-3[2,1]}=-1 / 2, \quad g_{3[2,2]}^{-3[-2,-1]}=g_{3[2,2]}^{-3[1,2]}=1 / 2,
$$

those of the matrix $g_{3[2,2]}^{-2}$ are

$$
g_{3[2,2]}^{-2[2,0]}=-\sqrt{1 / 2}, \quad g_{3[2,2]}^{-2[0,2]}=\sqrt{1 / 2},
$$

those of the matrix $g_{3[2,2]}^{-1}$ are

$$
\begin{aligned}
g_{3[2,2]}^{-1[0,1]} & =-\sqrt{1 / 5}, & g_{3[2,2]}^{-1[1,0]} & =\sqrt{1 / 5}, \\
g_{3[2,2]}^{-1[-1,-2]} & =g_{3[2,2]}^{-1[1,2]}=-\sqrt{3 / 20}, & g_{3[2,2]}^{-1[-2,-1]} & =g_{3[2,2]}^{-1[2,1]}=\sqrt{3 / 20},
\end{aligned}
$$

those of the matrix $g_{3[2,2]}^{0}$ are

$$
\begin{array}{ll}
g_{3[2,2]}^{0[1,-1]}=-\sqrt{2 / 5}, & g_{3[2,2]}^{0[-1,1]}=\sqrt{2 / 5}, \\
g_{3[2,2]}^{0[-2,2]}=-\sqrt{1 / 10}, & g_{3[2,2]}^{0[2,-2]}=\sqrt{1 / 10}
\end{array}
$$

those of the matrix $g_{3[2,2]}^{1}$ are

$$
\begin{array}{ll}
g_{3[2,2]}^{1[-1,0]}=-\sqrt{1 / 5}, & g_{3[2,2]}^{1[0,1]}=\sqrt{1 / 5}, \\
g_{3[2,2]}^{1[-2,1]}=g_{3[2,2]}^{1[-1,2]}=-\sqrt{3 / 20}, & g_{3[2,2]}^{1[1,-2]}=g_{3[2,2]}^{1[2,-11]}=\sqrt{3 / 20},
\end{array}
$$

and those of the matrix $g_{3[2,2]}^{2}$ are

$$
g_{3[2,2]}^{2[-2,0]}=-\sqrt{1 / 2}, \quad g_{3[2,2]}^{2[0,-22]}=\sqrt{1 / 2} .
$$

Finally, the nonzero elements of the Godunov-Gordienko matrix $g_{3[2,2]}^{3}$ are

$$
g_{3[2,2]}^{3[-1,2]}=g_{3[2,2]}^{3[1,-2]}=-1 / 2, \quad g_{3[2,2]}^{3[-2,1]}=g_{3[2,2]}^{3[2,-1]}=1 / 2 .
$$

We see that the Godunov-Gordienko matrices $g_{3[2,2]}^{t},-3 \leq t \leq 3$, are skewsymmetric. We have $B_{2(2,2)}(\|\boldsymbol{x}\|)=0$ by the same reasons as before.

Let $(n, q)=(2,2) \in A_{4}$. The corresponding term is

$$
\begin{aligned}
C_{224}^{i j \ell m}(\boldsymbol{x}) & =\sum_{r=-2}^{2} \sum_{s=-2}^{2} g_{2[1,1]}^{r[i, j]} g_{2[1,1]}^{s[\ell, m]} \sum_{t=-4}^{4} g_{4[2,2]}^{t[r, s]} U_{t 0}^{4}\left(k_{x}\right) B_{4(2,2)}(\|\boldsymbol{x}\|) \\
& =M_{i j \ell m}^{5}(\boldsymbol{x}) K_{5}(\|\boldsymbol{x}\|)
\end{aligned}
$$

with $K_{5}(\|\boldsymbol{x}\|)=B_{4(2,2)}(\|\boldsymbol{x}\|)$.

The nonzero elements of the Godunov-Gordienko matrices $g_{4[2,2]}^{ \pm 4}$ are

$$
g_{4[2,2]}^{-4[-2,2]}=g_{4[2,2]}^{-4[2,-2]}=g_{4[2,2]}^{4[2,2]}=-\sqrt{1 / 2}, \quad g_{4[2,2]}^{4[-2,-2]}=\sqrt{1 / 2},
$$


those of the matrices $g_{4[2,2]}^{ \pm 3}$ are

$$
\begin{aligned}
& g_{4[2,2]}^{-3[-2,1]}=g_{4[2,2]}^{-3[1,-2]}=g_{4[2,2]}^{-3[-1,2]}=g_{4[2,2]}^{-3[2,-1]}=g_{4[2,2]}^{3[1,2]}=g_{4[2,2]}^{3[2,1]}=-1 / 2, \\
& g_{4[2,2]}^{3[-2,-1]}=g_{4[2,2]}^{3[-1,-2]}=1 / 2,
\end{aligned}
$$

those of the matrices $g_{4[2,2]}^{ \pm 2}$ are

$$
\begin{aligned}
& g_{4[2,2]}^{-2[-1,1]}=g_{4[2,2]}^{-2[1,-1]}=g_{4[2,2]}^{2[1,1]}=-\sqrt{2 / 7}, \\
& g_{4[2,2]}^{-2[-2,0]}=g_{4[2,2]}^{-2[0,-2]}=g_{4[2,2]}^{2[0,2]}=g_{4[2,2]}^{2[2,0]}=\sqrt{3 / 14}, \\
& g_{4[2,2]}^{2[-1,-1]}=\sqrt{2 / 7},
\end{aligned}
$$

and those of the matrices $g_{4[2,2]}^{ \pm 1}$ are

$$
\begin{aligned}
& g_{4[2,2]}^{-1[-1,2]}=g_{4[2,2]}^{-1[2,-1]}=-1 /(2 \sqrt{7}), \\
& g_{4[2,2]}^{-1[-2,1]}=g_{4[2,2]}^{-1[1,-2]}=g_{4[2,2]}^{1[-2,-1]}=g_{4[2,2]}^{1[-1,-2]}=g_{4[2,2]}^{1[1,2]}=g_{4[2,2]}^{1[2,1]}=1 /(2 \sqrt{7}), \\
& g_{4[2,2]}^{-1[-1,0]}=g_{4[2,2]}^{-1[0,-1]}=g_{4[2,2]}^{1[0,1]}=g_{4[2,2]}^{1[1,0]}=\sqrt{3 / 7} .
\end{aligned}
$$

Finally, the nonzero elements of the Godunov-Gordienko matrix $g_{4[2,2]}^{0}$ are

$$
\begin{aligned}
g_{4[2,2]}^{0[-1,-1]} & =g_{4[2,2]}^{0[1,1]}=-\sqrt{8 / 35}, \\
g_{4[2,2]}^{0[-2,-2]} & =g_{4[2,2]}^{0[2,2]}=\sqrt{1 / 70}, \\
g_{4[2,2]}^{0[0,0]} & =6 / \sqrt{70} .
\end{aligned}
$$

Proof of (3-4). The first equation is obvious. The second and fourth equations may be checked by brute force, using the values of the matrix entries and GodunovGordienko coefficients calculated above. To prove the third equation, substitute (A.7) into (3-1). For the fifth equation, put $i=j=0$ and $m=p=2$ in (2-10). We have

$$
U_{r 0}^{2}\left(k_{\boldsymbol{x}}\right) U_{s 0}^{2}\left(k_{\boldsymbol{x}}\right)=\sum_{t=0}^{4} \sum_{v=-t}^{t} \sum_{w=-t}^{t} g_{t[2,2]}^{v[r, s]} U_{v w}^{t}\left(k_{\boldsymbol{x}}\right) g_{t[2,2]}^{w[0,0]}
$$

Using the matrix entries calculated above and the Godunov-Gordienko coefficients, we may rewrite this formula as

$$
U_{r 0}^{2}\left(k_{x}\right) U_{s 0}^{2}\left(k_{x}\right)=\frac{1}{5} \delta_{r s}+\sqrt{2 / 7} \sum_{t=-2}^{2} g_{2[2,2]}^{t[r, s]} U_{t 0}^{2}\left(k_{x}\right)+\frac{3 \sqrt{2}}{\sqrt{35}} \sum_{t=-4}^{4} g_{4[2,2]}^{t[r, s]} U_{t 0}^{4}\left(k_{x}\right) .
$$


Move all terms but the last from the right-hand side to the left-hand side, and substitute the result into the fifth line in (3-1). We obtain

$$
\begin{aligned}
M_{i j \ell m}^{5}(\boldsymbol{x})=\frac{\sqrt{35}}{3 \sqrt{2}} \sum_{n=-2}^{2} \sum_{p=-2}^{2} g_{2[1,1]}^{n[i, j]} g_{2[1,1]}^{p[\ell, m]}\left(U_{r 0}^{2}\left(k_{\boldsymbol{x}}\right) U_{s 0}^{2}\left(k_{\boldsymbol{x}}\right)-\frac{1}{5} \delta_{n p}\right. \\
\left.-\sqrt{2 / 7} \sum_{t=-2}^{2} g_{2[2,2]}^{t[r, s]} U_{t 0}^{2}\left(k_{\boldsymbol{x}}\right)\right) .
\end{aligned}
$$

Using (A.7) and (3-1), we get

$$
\begin{aligned}
M_{i j \ell m}^{5}(\boldsymbol{x})=\frac{\sqrt{35}}{3 \sqrt{2}}\left(\frac{3}{2} L_{i j \ell m}^{5}(\boldsymbol{x})-\frac{1}{2} L_{i j \ell m}^{4}(\boldsymbol{x})+\frac{1}{6} L_{i j \ell m}^{1}(\boldsymbol{x})\right) & \\
& -\frac{\sqrt{7}}{3 \sqrt{2}} M_{i j \ell m}^{2}(\boldsymbol{x})-\frac{\sqrt{5}}{3} M_{i j \ell m}^{4}(\boldsymbol{x}) .
\end{aligned}
$$

We finish the proof by using the second and fourth equations.

\section{References}

[Adams 1969] J. F. Adams, Lectures on Lie groups, W. A. Benjamin, New York, 1969.

[Boehler et al. 1994] J.-P. Boehler, A. A. Kirillov, Jr., and E. T. Onat, "On the polynomial invariants of the elasticity tensor", J. Elasticity 34:2 (1994), 97-110.

[Christakos 2005] G. Christakos, Random field models in earth sciences, Dover, Mineola, NY, 2005.

[Cressie 1993] N. A. C. Cressie, Statistics for spatial data, Wiley, New York, 1993.

[Godunov and Gordienko 2004] S. K. Godunov and V. M. Gordienko, “Коэффициенты КлебшаГордана при различных выборах базисов унитарных и ортогональных представлений групп SU(2), SO(3)", Sibirsk. Mat. Zh. 45:3 (2004), 540-557. Translated as "The ClebschGordan coefficients with respect to various bases for unitary and orthogonal representations of the groups SU(2) and SO(3)" in Sib. Math. J. 45:3 (2004), 443-458.

[Gordienko 2002] V. M. Gordienko, “Матричные элементы вещественных представлений групп $O(3)$ и SO(3)", Sibirsk. Mat. Zh. 43:1 (2002), 51-63. Translated as "Matrix entries of real representations of the groups $O(3)$ and $\mathrm{SO}(3)$ " in Sib. Math. J. 43:1 (2002), 36-46.

[Leonenko and Sakhno 2012] N. Leonenko and L. Sakhno, "On spectral representations of tensor random fields on the sphere”, Stoch. Anal. Appl. 30:1 (2012), 44-66.

[Lomakin 1964] V. A. Lomakin, "Statistical description of the stressed state of a body under deformation”, Dokl. Akad. Nauk SSSR 155:6 (1964), 1274-1277. In Russian.

[Malyarenko 2013] A. Malyarenko, Invariant random fields on spaces with a group action, Springer, Heidelberg, 2013.

[Marinucci and Peccati 2011] D. Marinucci and G. Peccati, Random fields on the sphere: representation, limit theorems and cosmological applications, London Mathematical Society Lecture Note Series 389, Cambridge University Press, 2011.

[Mathworks 2013] Symbolic math toolbox: user's guide, The Mathworks, Natick, MA, 2013, Available at http://www.mathworks.com/help/pdf_doc/symbolic/symbolic_tb.pdf. 
[Monin and Yaglom 1965] A. S. Monin and A. M. Yaglom, Статистическая гидромеханика: механика турбулентности, vol. 2, Nauka, Moscow, 1965. Translated as Statistical fluid mechanics, Volume 2, Dover, Mineola, NY, 2007.

[Ostoja-Starzewski 2008] M. Ostoja-Starzewski, Microstructural randomness and scaling in mechanics of materials, Chapman \& Hall/CRC, Boca Raton, FL, 2008.

[Ostoja-Starzewski et al. 2013] M. Ostoja-Starzewski, L. Shen, and A. Malyarenko, "Tensor random fields in conductivity and classical or microcontinuum theories", Math. Mech. Solids (2013).

[Porcu et al. 2012] E. Porcu, J.-M. Montero, and M. Schlather, Challenges in space-time modelling of natural events, Springer, Berlin, 2012.

[Robertson 1940] H. P. Robertson, "The invariant theory of isotropic turbulence", Proc. Cambridge Philos. Soc. 36 (1940), 209-223.

[Sena et al. 2013] M. P. Sena, M. Ostoja-Starzewski, and L. Costa, "Stiffness tensor random fields through upscaling of planar random materials", Probab. Eng. Mech. (2013).

[Spencer 1971] A. J. M. Spencer, "Theory of invariants”, pp. 239-353 in Continuum physics, vol. 1, edited by A. C. Eringen, Academic Press, New York, 1971.

[Varshalovich et al. 1975] D. A. Varshalovich, A. N. Moskalev, and V. K. Khersonskiй, Квантовая теория углового момента: аппарат неприводимых тензоров, сферические функции, 3nj-симболы, Nauka, Leningrad, 1975. Translated as Quantum theory of angular momentum: irreducible tensors, spherical harmonics, vector coupling coefficients, 3 nj symbols, World Scientific, Teaneck, NJ, 1988.

[Yaglom 1957] A. M. Yaglom, "Some classes of random fields in $n$-dimensional space, related to stationary stochastic processes", Theor. Probab. Appl. 2:3 (1957), 273-320.

Received 12 Feb 2013. Revised 15 Jul 2013. Accepted 11 Sep 2013.

ANATOLIY MALYARENKO: anatoliy.malyarenko@mdh.se

Division of Applied Mathematics, Mälardalen University, Box 883, Högskoleplan 1,

SE-721 23 Västerås, Sweden

MARTIN OSTOJA-STARZEWSKI: martinos@illinois.edu

Department of Mechanical Science and Engineering, University of Illinois at Urbana-Champaign, 1206 W. Green Street, Urbana, IL 61801-2906, United States 


\section{EDITORIAL BOARD}

ANTONIO CARCATERRA ERIC A. CARLEN

FRANCESCO DELL'ISOLA

RAFFAELE ESPOSITO

ALBERT FANNJIANG

Gilles A. FrancFort

PiERANGElo MARCATI

JEAN-JACQUES MARIGO

PETER A. MARKOWICH MARTIN OSTOJA-STARZEWSKI

PIERRE SEPPECHER

DAVID J. STEIGMANN

PAUl STEINMANN

PierRe M. Suquet

\section{MANAGING EDITORS}

MICOL AMAR

CORRADO LATTANZIO

ANGELA MADEO

MARTIN OSTOJA-STARZEWSKI

\section{ADVISORY BOARD}

ADNAN AKAY

Holm AltenbaCH

MICOL AMAR

HARM ASKES

TEODOR ATANACKOVIĆ

VICTOR BERDICHEVSKY

GuY BouchitTÉ

ANDREA BRAIDES

ROBERTO CAMASSA

MAURO CARFORE

ERIC DARVE

FELIX DARVE

ANNA DE MASI

Gianpietro Del Piero

EMMANUELE Di BENEDETTO

BERNOLD FIEDLER

IRENE M. GAMBA

SERGEY GAVRILYUK

TIMOTHY J. HEALEY

DOMINIQUE JEULIN

ROGER E. KHAYAT

CORRADO LATTANZIO

ROBERT P. LIPTON

ANGELO LUONGO

ANGEla MadeO

JUAN J. MANFREDI

CARLO MARCHIORO

GÉrard A. MAUGin

ROBERTO NATALINI

PATRIZIO NEFF

ANDREY PIATNITSKI

ERrico Presutti

MARIO PUlVIRENTI

LuCiO RuSSO

Miguel A. F. SANJUAN

Patrick SElvadurai

ALEXANDER P. SEYRANIAN

MIROSLAV ŠILHAVÝ

GUIDO SWEERS

ANTOINETTE TORDESILLAS

LEV TRUSKINOVSKY

JUAN J. L. VELÁZQUEZ

VINCENZO VESPRI

ANGELO VULPIANI msp.org/memocs

Università di Roma “La Sapienza”, Italia

Rutgers University, USA

(CO-CHAIR) Università di Roma "La Sapienza", Italia

(TREASURER) Università dell'Aquila, Italia

University of California at Davis, USA

(CO-CHAIR) Université Paris-Nord, France

Università dell' Aquila, Italy

École Polytechnique, France

DAMTP Cambridge, UK, and University of Vienna, Austria

(CHAIR MANAGING EDITOR) Univ. of Illinois at Urbana-Champaign, USA

Université du Sud Toulon-Var, France

University of California at Berkeley, USA

Universität Erlangen-Nürnberg, Germany

LMA CNRS Marseille, France

Università di Roma “La Sapienza”, Italia

Università dell' Aquila, Italy

Université de Lyon-INSA (Institut National des Sciences Appliquées), France (CHAIR MANAGING EDITOR) Univ. of Illinois at Urbana-Champaign, USA

Carnegie Mellon University, USA, and Bilkent University, Turkey

Otto-von-Guericke-Universität Magdeburg, Germany

Università di Roma "La Sapienza”, Italia

University of Sheffield, UK

University of Novi Sad, Serbia

Wayne State University, USA

Université du Sud Toulon-Var, France

Università di Roma Tor Vergata, Italia

University of North Carolina at Chapel Hill, USA

Università di Pavia, Italia

Stanford University, USA

Institut Polytechnique de Grenoble, France

Università dell'Aquila, Italia

Università di Ferrara and International Research Center MEMOCS, Italia

Vanderbilt University, USA

Freie Universität Berlin, Germany

University of Texas at Austin, USA

Université Aix-Marseille, France

Cornell University, USA

École des Mines, France

University of Western Ontario, Canada

Università dell' Aquila, Italy

Louisiana State University, USA

Università dell' Aquila, Italia

Université de Lyon-INSA (Institut National des Sciences Appliquées), France

University of Pittsburgh, USA

Università di Roma "La Sapienza", Italia

Université Paris VI, France

Istituto per le Applicazioni del Calcolo "M. Picone", Italy

Universität Duisburg-Essen, Germany

Narvik University College, Norway, Russia

Università di Roma Tor Vergata, Italy

Università di Roma "La Sapienza", Italia

Università di Roma “Tor Vergata", Italia

Universidad Rey Juan Carlos, Madrid, Spain

McGill University, Canada

Moscow State Lomonosov University, Russia

Academy of Sciences of the Czech Republic

Universität zu Köln, Germany

University of Melbourne, Australia

École Polytechnique, France

Bonn University, Germany

Università di Firenze, Italia

Università di Roma La Sapienza, Italia

MEMOCS (ISSN 2325-3444 electronic, 2326-7186 printed) is a journal of the International Research Center for the Mathematics and Mechanics of Complex Systems at the Università dell'Aquila, Italy.

Cover image: "Tangle" by @ John Horigan; produced using the Context Free program (contextfreeart.org).

\section{PUBLISHED BY}

mathematical sciences publishers

nonprofit scientific publishing

http://msp.org/

(C) 2014 Mathematical Sciences Publishers 
Mathematics and Mechanics of Complex Systems vol. 2 no. 2

A mixed boundary value problem in potential theory for a bimaterial porous region: An application in the environmental geosciences

A. P. S. Selvadurai

Geometric degree of nonconservativity

Jean Lerbet, Marwa Aldowaji, Noël Challamel, Oleg N.

Kirillov, François Nicot and Félix Darve

Asymptotic analysis of small defects near a singular point in antiplane elasticity, with an application to the nucleation of a crack at a notch

Thi Bach Tuyet Dang, Laurence Halpern and Jean-Jacques Marigo

The homogenized behavior of unidirectional fiber-reinforced composite materials in the case of debonded fibers

Yahya Berrehili and Jean-Jacques Marigo

Statistically isotropic tensor random fields: Correlation structures

Anatoliy Malyarenko and Martin Ostoja-Starzewski

MEMOCS is a journal of the International Research Center for the Mathematics and Mechanics of Complex Systems at the Università dell' Aquila, Italy.

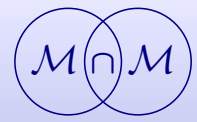

